\title{
Meandering of a particle-laden rivulet
}

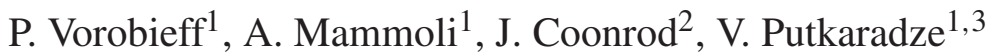 \\ \& K. Mertens ${ }^{4}$ \\ ${ }^{1}$ Department of Mechanical Engineering, The University of New Mexico, \\ USA \\ ${ }^{2}$ Department of Civil Engineering, The University of New Mexico, USA \\ ${ }^{3}$ Department of Mathematics, Colorado State University, USA \\ ${ }^{4}$ Carolina Center for Interdisciplinary Applied Mathematics, \\ The University of North Carolina, USA
}

\begin{abstract}
The behavior of a rivulet flowing down an incline is a fundamental problem in hydrodynamics, with many important applications in water resources engineering, largely because of its connection with natural meandering flows (rivers and streams). Recent advances in the understanding of laboratory rivulet flows reveal several important features of the rivulet behavior that are directly relevant to this connection with natural flows. Rivulet meandering is triggered by irregularities in the flow rate at the origin, and amplified by re-absorption of droplets left on the inclined surface by the previous meanderings. This leads to a statistically nontrivial behavior, with the spectrum of an ensemble of rivulet deviations from its centreline obeying a power law. Some of the statistics of the laboratory rivulets (e.g., the area swept by the rivulet) closely resemble those of real rivers (Hack's law). However, there are many important physical differences between rivulets and real rivers. Among them is the fact that the flow in real rivers and streams is often multiphase (sediment-laden). Here we present the results of laboratory experiments with rivulets, where the flow down an inclined, partially wetting plane carries a well-characterized particulate to model sediment flow. The most notable change produced by the addition of the particles is the formation of a stationary meandering pattern, which does not occur under the same conditions for the flow without particles.

Keywords: meandering, free-surface flow, flow with particles, rivulet.
\end{abstract}




\section{Introduction}

Rivers and streams meander, with their paths gradually changing, leaving behind crescent lakes, eroding the shores [1], transporting sediment, and in the process changing and defining the shape of Earth's surface (Fig. 1a). Some of the most famous natural monuments (such as the Grand Canyon) owe their existence to river meandering [2]. Flow of ancient rivers is likely responsible for some very prominent features on the surface of Mars ([3], also see Fig. 1b). As recently revealed by the Cassini/Huygens probe, rivers also flow and meander on Titan [4], the satellite of Saturn, although it is liquid methane, not water, that flows there between the shores of ice at a chilly 93 Kelvin (Fig. 1c).

Meandering is affected by many complex factors, including turbulence in the water, erosion of the soil on the bottom, unevenness of the soil properties, and variations in flow rates due to the seasons. However, there are some simple statistical properties that apply to all rivers and streams. The most notable of them is Hack's law, which defines a relationship between the length of a stream or river $L$ and the size of its drainage basin $A$ (the area of land where water from rain and snow melt drains downhill into the river). This law was discovered by an American geophysicist J.T. Hack in 1957 [5]. Hack studied the streams of the Shenandoah Valley and adjacent mountains in Virginia, and obtained a power-law formula $L=1.4 A^{h}$, where $h$ (Hack's exponent) is a constant value which turns out to be the same or almost the same for Shenandoah Valley and for several thousands of other river basins for which measurements were made. The most common value of $h$ is about 0.57 .

It is notoriously hard to answer exactly which of the multitude of the possibly important and highly intertwined geological and meteorological factors contributes most to the river meandering. As in many other cases where there is an interesting natural phenomenon which is both too complicated and too big to fit in a lab on a modest budget, physicists came up with an experimental model of gravitydriven meandering flow - a rivulet on an inclined plane. This model can be easily reproduced in a kitchen sink by placing a tilted cookie sheet under a slightly opened faucet. In this kitchen-sink experiment, the tiny rivulet will meander, producing a curious pattern not unlike a miniature river, although in a properly maintained kitchen, there should be no precipitation, erosion, or sedimentation interfering with the flow. Similar observations on somewhat larger sheets in laboratory led to the conclusion that, since the rivulet always meanders, there must be some inherent instability that causes it to behave this way. It was also commonly thought that meandering of a rivulet has some kind of a most frequently occurring spatial scale (called characteristic wavelength $\lambda$ ) associated with it.

However, the basic premise of many rivulet studies - that the rivulet will always meander - was recently demonstrated to be incorrect, with perfectly straight, stable rivulets (Fig. 1d) obtained experimentally [6,7]. The key element in stabilizing the rivulets is the flow rate. It has to be maintained at a constant value, free of any fluctuations. A discharge through a small hole in the bottom of a very tall reservoir would have such fluctuation-free quality; a discharge from a kitchen faucet would 

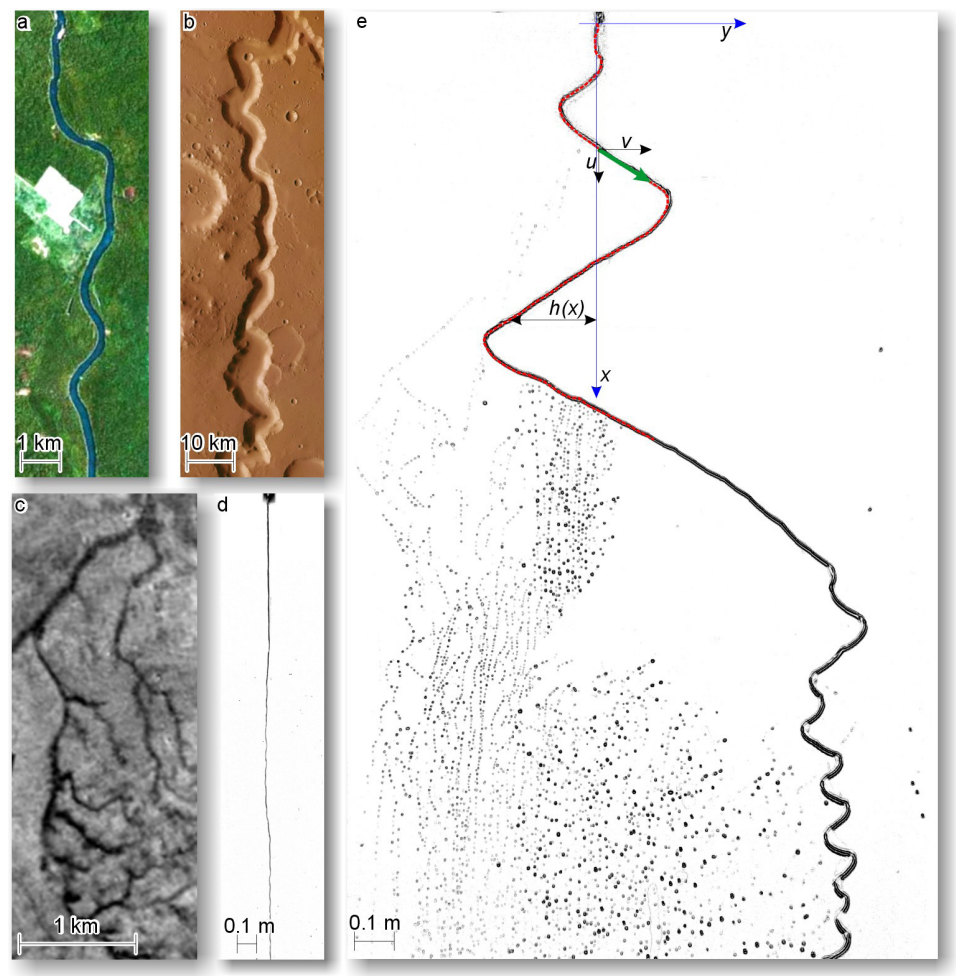

Figure 1: Natural (a-c) and laboratory (d-e) flows, view from above, flow direction is from top to bottom where known. a, Flow of Machadinho river, Brazil. The image extent is about $12 \mathrm{~km}$. Source: Google Earth/TerraMetrics. b, Mars Express image of Nanedi Valles on Mars captured on October 3, 2004. Source: European Space Agency (ESA)/DLR/FU Berlin (G. Neukum). The image extent is about $100 \mathrm{~km}$. c, Cassini/Huygens image of a river channel on Titan. The image extent is about $4 \mathrm{~km}$. Source: ESA/NASA/JPL/University of Arizona. d, Non-meandering flow (constant flow rate) of a $50 \%-50 \%$ water-glycerine mix on acrylic substrate. e, Meandering flow under the same conditions, with flow rate fluctuations induced by a pulsed electromagnetically operated valve. The overlay shows the centerline (gray dashed line), the coordinate system, and the velocity components. The image extent in $\mathrm{d}$ and e is $2.4 \mathrm{~m}$.

not. In most natural and laboratory flows, the flow rate will fluctuate, and thus the stream will meander. Remove the fluctuations though, and you get a stable rivulet, sometimes forming a pretty braiding pattern explained in our earlier work with a simple theoretical model [7]. This study, however, did not attempt to provide a similar explanation for the meandering flow regime that would emerge in the case the flow rate was not constant. 


\section{Meandering rivulet studies}

In our subsequent experiments with a specially built inclined plane apparatus, we tried several ways of perturbing the flow. We found that the best way to produce meandering was to add an electronically controlled solenoid valve to the discharge tube feeding fluid from the tall reservoir to the incline. With this valve squeezing the tube every few seconds, the flow would start to meander (Fig. 1e). With the valve turned off, it would gradually straighten out.

We took several hundred pictures of the meandering rivulet and started analyzing their statistics. We assembled a plot showing how often each meandering frequency $f$, and corresponding length scale $l=1 / f$, show up in the picture database. This plot (a power spectrum) revealed that for a wavenumber $k=2 \pi / l$, the corresponding value of the power spectrum $S(k)$ was proportional to $k^{-5 / 2}$. This relationship (a power law) holds in a range from the characteristic stream width (about $5 \mathrm{~mm}$ ) to the largest scale we can measure $(2.4 \mathrm{~m}$, the extent of our inclined plane). Now, if a characteristic wavelength $\lambda$ existed, it would correspond to a characteristic wavenumber $k_{\max }=2 \pi / \lambda$, where the spectrum would peak. But the spectra we measure show no such peaks, in contradiction with what earlier works had suggested.

Power-law statistics are Nature's way of telling us that something simple and profound is happening. Thus we tried to explain the behavior of the stream in our experiment with a model that only takes into consideration the most basic features of our flow. We averaged out velocity variations in the stream cross-section. We replaced the actual (variable) value of the contact angle between the fluid, the air, and the surface, with another average [8]. Finally, we radically simplified one very important feature of the stream. A real meandering stream on a partially wetting plane sheds droplets as it changes its course, like a meandering river leaving crescent lakes in its old path (of course, in the latter case, the process is much more complex). These droplets may get reabsorbed into the rivulet during subsequent meanderings. The physics of each such event are rather complex, but, instead of trying to model it exactly, we account for such stream-droplet interactions by adding a random forcing term to our equations. This term has to be statistically faithful to what we see in experiment, but it is much simpler to deal with. We derived two equations (for the two coordinates in the plane) describing the fluid moving due to a combination of surface tension, gravity, shear in the flow near the surface (lubrication approximation), viscous dissipation inside the fluid, and random forcing due to droplets. We added the law of conservation of mass (in the form of a continuity equation). Thus we obtained a simple system that could be quickly solved numerically using an ordinary personal computer. This model system can produce rivulet shapes in the plane that are very similar to those we saw in experiment, and with the same statistical properties.

We repeated our experiments on several surfaces made of different materials, with different static contact angles. The higher the contact angle, the easier it is for a droplet to roll off the surface. Fewer droplets mean weaker random forcing, and that reflects in a slower growth of the meandering amplitude, again in nice 
agreement with our theory [9]. However, on each surface we studied, the scaling of the power spectrum was the same: $S(k) \sim k^{-5 / 2}$. Working with different surfaces, we found another curious thing. A real river has a drainage basin. Our rivulet also has a drainage basin of a sort - it can encounter droplets left by the previous meanderings, and these droplets are typically confined between the rivulet path and the rivulet's initial course before we began perturbing the flow (the straight line). What turned out is that the area of this "drainage basin" obeys the same scaling law as the drainage basin of a real river - Hack's law! Moreover, our theory predicts how the value of Hack's law exponent is related to the scaling of the meandering power spectrum. If that scaling is $-5 / 2$, then Hack's law exponent should be 4/7 [8]. Surprisingly, $4 / 7$ approximately equals 0.57 , the same value we see in laboratory rivulets and in real rivers.

\section{Observations of stationary meandering}

One of the notable differences between our work and some of the earlier experiments lay in the rarity of observed stream pinning events. Pinning (or stationary meandering) occurs when a meandering flow pattern ceases to change with time. While it was reported to occur fairly commonly by other researchers [10], in our experiments pinning was rare and could always be attributed to the inclined plane being contaminated with dust or dried-up residue from earlier experiments. With the incline wiped clean and the experiment restarted, pinning did not re-occur. Meandering would persist for as long as the flow rate was perturbed by the solenoid valve. If the flow rate perturbation ceased, the flow would gradually transition to a straight rivulet stationary state.

Unlike our laboratory rivulet, natural flows are always multi-phase to some extent, carrying sediments, organic matter (e.g., dead skunks), and debris. As the flow rate changes in an alluvial system, scour or deposition will occur, further affecting meandering patterns [11]. Sediment transport was believed to be necessary for flow meanders [12], however, experiments without sediment transport also manifest meandering $[8,10,13]$. While the notion of meandering as an inherent global flow instability that arose in the 1980s [13] has been experimentally disproven $[6,7]$, transverse shear stress distribution along the wetted perimeter of the meandering stream [14] likely still plays a role in meandering. It is also notable that, in the case of natural flows, their course can also persist for a long duration. This persistence can be aided by erosion of the riverbed. On the other hand, strong (catastrophic) changes in the natural flow rate can lead to changes in the established course of the stream. Thus we attempted to bring our experiment one step closer to these natural flows by adding a particulate phase to the fluid. In the experiments described here, the fluid was Albuquerque tap water (with small naturally occurring salinization), and we added a particulate phase to it. The latter was comprised of small, nearly monodisperse $(500 \pm 75 \mu \mathrm{m})$, and almost neutrally buoyant (specific gravity $\sim 1.05$ ) polystyrene microspheres, in fractions that were varied from 0.5 to $5 \%$ of the total volume of the flow. The water-particle mixture was driven into the tall reservoir by a peristaltic pump from a reservoir 
that collected the runoff from the bottom of the inclined plane. On the time scale characterizing the experiment, settling of the particles in the top reservoir was negligible.

The resulting behavior of the multiphase meandering flow (Fig. 2) turned out to be quite interesting. There was no qualitative difference between the pure water flow and the flow of the water with particles when the flow rate was periodically disrupted by the solenoid valve (flow reduction by $25 \%$ for $0.1 \mathrm{~s}$ every $2 \mathrm{~s}$ ) - in both cases, the flow continued to meander. The difference emerged, however, when the valve was turned off. The pure water flow in that case eventually reverted to a straight rivulet, with relaxation times between 40 minutes and 2.5 hours. Under the same conditions, the flow with particles simply retained its course for as long as the experiment was run (up to 4 hours).

\section{Discussion and future work}

Consider a stationary bend in the rivulet. It doesn't have to be stationary in the strict sense, just stable enough to persist on the time scale of interest (several minutes). For this bend, the hydrodynamic centrifugal force is balanced by the pressure gradient. At least, this should be the case for the flow of a pure fluid. However, if a heavy (non-neutrally buoyant) particle is present in the flow, it will be subject to the centrifugal force as it passes the bend.

This particle can cause pinning if it gets trapped near the edge of the rivulet as shown in Fig. 3, A-A. The unbalanced force acting upon a particle of radius $a$ is

$$
F=\Delta \rho a^{3} \frac{U^{2}}{R},
$$

where $\Delta \rho$ is the density difference between the particle and the fluid, $U$ is the flow velocity and $R$ is the radius of curvature of the bend. Drift velocity towards the edge can be estimated as

$$
v=\frac{F}{6 \pi \rho a v}=\frac{\Delta \rho}{\rho} \frac{a^{2}}{6 \pi v} \frac{U^{2}}{R},
$$

where the denominator represents Stokes drag on the particle. Sedimentation near the edge happens if $v \tau \leq d$, where $\tau=L / U$ is the time it takes for the flow to travel the length of the bend, and $d$ is the characteristic cross-stream distance. Assuming $L \simeq \pi R$, which holds exactly if the bend is a semi-circle, the probability $p$ of sedimentation for a single particle traversing the bend is

$$
p=\frac{\Delta \rho}{\rho} \frac{a^{2} U}{6 v d} .
$$

For our flow, the characteristic values are as follows. $U \sim 1 \mathrm{~m} / \mathrm{s}, d \sim 0.01 \mathrm{~m}$, $\Delta \rho / \rho \simeq 0.05, v \sim 10^{-6} \mathrm{~m}^{2} / \mathrm{s}, a \simeq 5 \times 10^{-4} \mathrm{~m}$. Thus $p \sim 2 \times 10^{-3}$. Once a particle passes through the bend without being stuck it will move to next bend and 


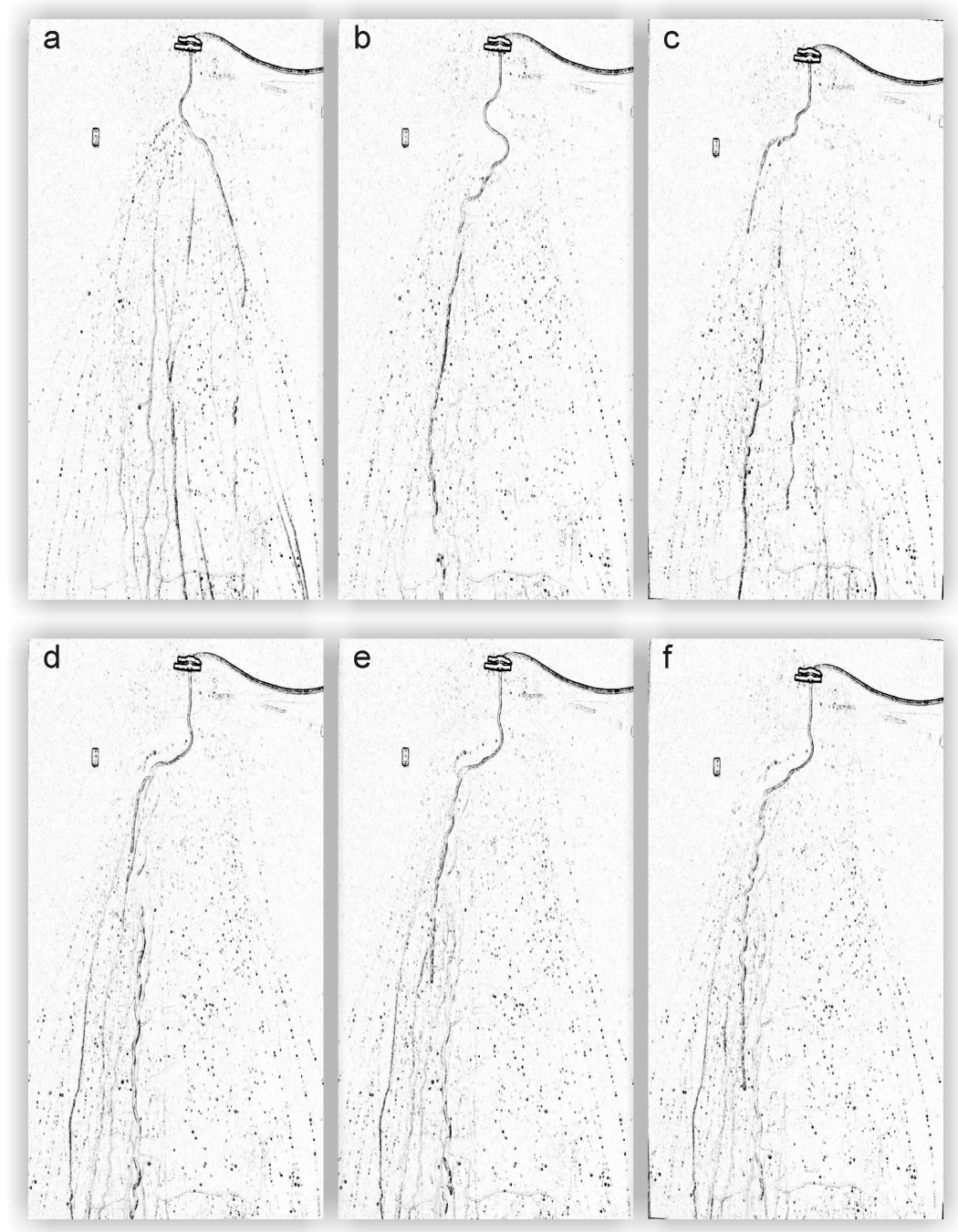

Figure 2: Snapshots of meandering multiphase flow. The flow direction is from top to bottom, the physical extent of the imaged area in this direction is $2.4 \mathrm{~m}$. The images are separated with an 8-second interval. In the first three images (top row, a-c), the flow is subjected to a periodic perturbation in the flow rate, causing continuous meandering. Between the third (c) and fourth (d) image, the flow rate perturbations cease, producing a stationary meandering pattern (bottom row, d-f). In the images shown, the flow is a mixture of $99 \%$ water with $1 \% 500$ micron polystyrene microspheres by volume, and the flow rate is about $100 \mathrm{~cm}^{3} / \mathrm{s}$. 

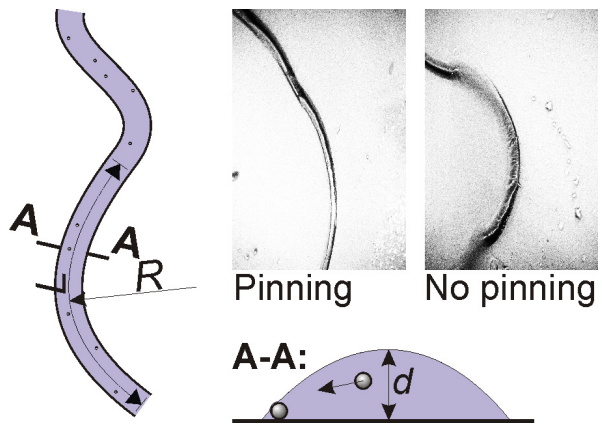

Figure 3: Left - view of a rivulet flow carrying particles through a bend. $L$ and $R$ are the characteristic length and radius of the bend. A-A (bottom center) shows a cross-section view of the rivulet, with $d$ being the characteristic cross-sectional size. Top center - photo close-up of a stationary meandering rivulet (note that the contact angles on both sides are the same). Top right - photo close-up of a transient (non-stationary) meandering rivulet with different advancing and receding contact angles.

so forth. An interesting feature of equation (1) is that the probability of being stuck at the bend does not depend on the bend's radius.

Let us now consider a more realistic situation when many particles are moving through the bend at the same time. Suppose the density of particles per unit volume is $\alpha$. Since the cross-section of the stream is of the order $d^{2}$, there particles are moving through the fluid bend with the rate

$$
n \simeq \alpha d^{2} U
$$

Thus, after the time $t, N=\alpha d^{2} U t$ particles will have passed through the bend. The probability of a single particle passing through the bend is $1-p$. The probability of $N$ independent particles passing through (assuming that the particles are spread far enough for each sticking event to be statistically independent) is $(1-p)^{N} \simeq e^{-N p}$. Thus the probability of at least one of $N$ particles sticking $p_{s}$ as they go through is

$$
p_{s}=1-e^{-N p}=1-e^{-\alpha d^{2} U t p}=1-e^{t / T_{s}} .
$$

Again, interestingly enough, the probability of pinning is characterized by the flow parameters, but is not dependent on the bend's characteristics. We can thus define the time scale $T_{S}$ for particle pinning in a single bend as

$$
T_{p} \sim \frac{1}{\alpha d^{2} U p} .
$$

After the time $t \gg T_{p}$, the meandering trajectory of the rivulet will be frozen in time with probability being almost 1 . More precisely, if we define $T_{p}$ as the time 
after which the probability of meandering being frozen is, say, $90 \%$, then we get a more precise version of formula (3):

$$
T_{p}=\frac{\log 10}{\alpha d^{2} U p} .
$$

Consider the typical particle concentration producing pinning in our experiments $-5 \%$ by volume. This means that $1 \mathrm{~cm}^{3}$ contains about $\alpha=95$ particles with the radius of 500 microns. Assuming a characteristic cross-sectional stream area $d^{2} \sim 0.1 \mathrm{~cm}^{2}$, the volume of the flow traversing this area in one second is $10 \mathrm{~cm}^{3}$, about a thousand particles will be carried through each cross-section per second. Then, (4) gives $T_{s} \sim 1.2 \mathrm{sec}$, which explains a near-instantaneous pinning of the stream. While equation (4) is of course just a rough estimate, it is useful in evaluating the order of magnitude for the pinning time, which is clearly small. More detailed evaluation of the pinning time can be obtained by constructing a more detailed model of particle motion very close to the contact line, improving on a rather simple-minded version of the Stokes drift we have utilized here.

Note that pinning does not occur when the flow rate is subjected to fluctuations as described in the previous part. In the immediate future, we will study the sensitivity of a pinned (stationary) rivulet to flow-rate perturbations varied in duration, frequency, and intensity. While our simple model deliberately does not include many aspects of real streams (such as an erodible riverbed), the relationship between flow-rate fluctuations and the transition between stationary and non-stationary meandering can provide some insights into one important aspect of the behavior of natural rivers. Most of these rivers change their course somewhat infrequently, although their flow rates do fluctuate quite a bit. However, an unusually strong fluctuation (for example, due to a particularly intense rainfall) still can destabilize the course of a real river. With global climate change affecting the precipitation patterns throughout the world, any quantitative insights into what amount of fluctuation in the flow rate could cause a catastrophic change in the course of a natural stream would be very helpful.

\section{Acknowledgements}

This research is supported by the National Science Foundation New Mexico EPSCoR (Experimental Program to Stimulate Competitive Research) award EPS0447691. This work was also partially supported by the US Department of Energy (DOE). The financial support does not constitute an endorsement by the DOE of the views expressed in this paper

\section{References}

[1] Langbein, W. \& Leopold, L., River meanders - theory of minimum variance. U.S. Geological Survey Professional Paper 422-H, 1966. 
[2] Stevens, L., Schmidt, J., Ayers, T. \& Brown, B., Flow regulation, geomorphology, and Colorado River marsh development in the Grand Canyon, Arizona. Ecological Applications, 5(4), pp. 1025-1039, 1995.

[3] Malin, M. \& Edgett, K., Evidence for persistent flow and aqueous sedimentation on early Mars. Science, 302, pp. 1931-1934, 2003.

[4] C.C. Porco et al., Imaging of Titan from the Cassini spacecraft. Nature, 434, pp. 159-168, 2005.

[5] Hack, J., Studies of longitudinal stream profiles in Virginia and Maryland. U.S. Geological Survey Professional Paper 294-B, 1957.

[6] Mertens, K., Putkaradze, V. \& Vorobieff, P., Braiding patterns on an inclined plane. Nature, 430, p. 165, 2004.

[7] Mertens, K., Putkaradze, V. \& Vorobieff, P., Morphology of a stream flowing down an inclined plane. Part 1. Braiding. Journal of Fluid Mechanics, 531, pp. 49-58, 2005.

[8] Birinr, B., Mertens, K., Putkaradze, V. \& Vorobieff, P., Morphology of a stream flowing down an inclined plane. Part 2. Meandering. Journal of Fluid Mechanics, 607, pp. 401-411, 2008.

[9] Birinr, B., Mertens, K., Putkaradze, V. \& Vorobieff, P., Meandering fluid streams in the presence of flow-rate fluctuations. Physical Review Letters, 101, p. 114501, 2008.

[10] Le Grand-Piteira, N., Daerr, A. \& Limat, L., Meandering rivulets on a plane: A simple balance between inertia and capillarity. Physical Review Letters, 96, p. 254503, 2006.

[11] Engelund, F. \& Skovgaard, O., On the origin of meandering and braiding in alluvual streams. Journal of Fluid Mechanics, 57, pp. 289-302, 1973.

[12] Parker, G., On the cause and characteristic scales of meandering and braiding in rivers. Journal of Fluid Mechanics, 76, pp. 457-480, 1975.

[13] Nakagawa, T. \& Hotsuta, M., Note on boundary effects on stream meandering. Sedimentology, 31, pp. 119-122, 1984.

[14] Nakagawa, T., Boundary effects on stream meandering and river morphology. Sedimentology, 30, pp. 117-127, 1983. 